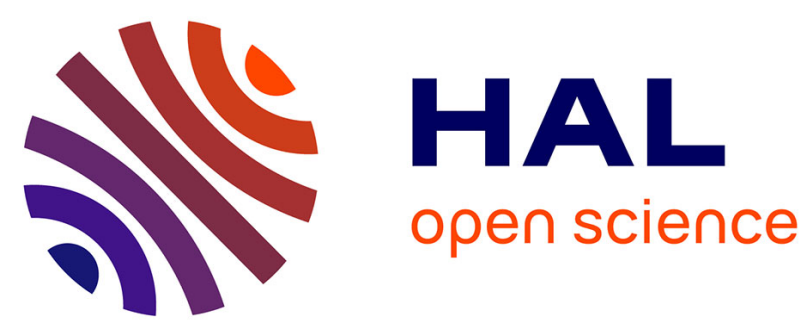

\title{
Identification in the Terahertz Domain using Low Cost Tags with a Fast Spectrometer
}

Maher Hamdi, Florent Bonnefoy, Maxime Bernier, Frédéric Garet, Etienne Perret, Nicolas Barbot, Romain Siragusa, David Hely

\section{To cite this version:}

Maher Hamdi, Florent Bonnefoy, Maxime Bernier, Frédéric Garet, Etienne Perret, et al.. Identification in the Terahertz Domain using Low Cost Tags with a Fast Spectrometer. ASID 2018: 12th IEEE International Conference on Anti-counterfeiting, Security, and Identification, Nov 2018, Xiamen, China. 10.1109/ICASID.2018.8693209 . hal-02015383

\section{HAL Id: hal-02015383 \\ https://hal.univ-grenoble-alpes.fr/hal-02015383}

Submitted on 5 Mar 2020

HAL is a multi-disciplinary open access archive for the deposit and dissemination of scientific research documents, whether they are published or not. The documents may come from teaching and research institutions in France or abroad, or from public or private research centers.
L'archive ouverte pluridisciplinaire HAL, est destinée au dépôt et à la diffusion de documents scientifiques de niveau recherche, publiés ou non, émanant des établissements d'enseignement et de recherche français ou étrangers, des laboratoires publics ou privés. 


\title{
Identification in the Terahertz Domain using Low Cost Tags with a Fast Spectrometer
}

\author{
Maher Hamdi ${ }^{1}$, Florent Bonnefoy ${ }^{2}$, Maxime Bernier ${ }^{2}$, Frédéric Garet ${ }^{2}$, Etienne Perret ${ }^{3}$, Nicolas Barbot ${ }^{3}$, Romain \\ Siragusa ${ }^{3}$, David Hely ${ }^{3}$ and Eiji Kato ${ }^{4}$ \\ ${ }^{1}$ DRT, CTRegCEA, ${ }^{1} 33600$ Pessac, France, \\ ${ }^{2}$ University of Savoie Mont Blanc, CNRS Grenoble INP, IMEP-LAHC, ${ }^{2} 73370$ Le Bourget du Lac, France \\ ${ }^{3}$ University Grenoble Alpes, LCIS, ${ }^{3} 26000$ Valence, France, \\ ${ }^{4}$ Advantest Corp, ${ }^{4}$ 48-2 Matsubara, Kamiayashi, Aoba-ku, Sendai 980-3124, Japan \\ 1e-mail: Maher.Hamdi@cea.fr
}

\begin{abstract}
In this paper, we report on fast identification of low-cost tags with a commercial terahertz (THz) spectrometer. These tags are designed and dedicated for anti-counterfeiting applications. One-dimensional pattern on a fully dielectric material were designed to enhance the robustness and complex the reverse engineering. Moreover, contrary to barcodes and RFID tags based on a planar surface pattern, this one is buried in the volume and can be then hidden in the product to identify as it is only made of dielectric material, without any metal. The generic tag consists of a quasi-periodic stack of two different polyethylene-based dielectric layers with different refractive indices and thicknesses. As we use THz wave, each layer remains thin (of the order of the wavelength), to get a tag whose thickness is in the millimeter range. We show that a reliable identification of the binary code encoded by the tag is possible in few tens of millisecond, with a bit error rate smaller than 1 percent.
\end{abstract}

Keywords- counterfeiting; fast identification; low-cost; RFID; THID tags; terahertz.

\section{INTRODUCTION}

The Identification in the Terahertz (THz) domain (THID) using tags based on structured materials is a promising way to address counterfeiting issues ${ }^{[1]-[2]}$. This technology is currently limited by the terahertz $(\mathrm{THz})$ reader developments in term of cost, compactness and acquisition speed, which remain the main crucial points for such applications. THID aims at proposing solutions to push the limits of identification technologies currently used. The specific properties of the materials in the $\mathrm{THz}$ frequency domain allow to consider a new generation of tags, in order to strengthen the protection of the data contained inside. The current technologies such as RFID and barcodes can be easily reverse-engineered and counterfeited ${ }^{[3]-[5]}$, since they are composed of printed schemes or metal parts on the top of the label. The first THID approach has been revealed by D. Cumming in 2003, who demonstrated the possibility to create a $\mathrm{THz}$ hologram embedded in a dielectric material ${ }^{[6]}$. In 2015, Y. Guan et al. have proposed structures based on polyethylene and carbon layers which are stacked ${ }^{[7]}$. Very recently, tags based on photonic-crystal slabs using silicon material were proposed as a large storage capacity of $48 \mathrm{bits} / \mathrm{cm}^{2}{ }^{[8]}$. Alternative THz tags structures based on diffractive gratings were proposed to address authentication and package damage inspection. ${ }^{[9]-[11]}$. The initial structure of the THID tag we proposed in this paper is a 1D periodic stack of dielectric layers whose overall thickness is in the millimeter range ${ }^{[12]}$. The frequency response (transmission and reflection) of such tag is ruled by the material and the geometrical parameters of each layer. Forbidden frequency bang gap (named Photonic Band Gap: PBG) appears in this response. Changing the structure of the stacking by especially modifying the thickness or the refractive index of one or several layers (named structural defect), the spectral response of the corresponding THID tag is also impacted. Indeed, when the periodicity of the structure is broken, some peaks appear in the PBG. The number and the spectral position of these peaks allows to encode an information in the tag which can be therefore used as reliable and unique identifier. In order to address real application issues, the $\mathrm{THz}$ reader must be able to measure the identifier stored in any tag in quasi real time. After introducing the tag structure, we will describe how we encode information buried in the tag and how many bits can be stored with such structures. Then, we will focus on the capacity of a currently available commercial $\mathrm{THz}$ spectrometer to perform reliable identification of these tags in a realistic duration for the targeted real-time applications.

\section{TAG DESCRIPTION}

As previously described, the proposed $\mathrm{THz}$ tag is made of a stack of two different transparent thin dielectric films exhibiting different refractive index in the $\mathrm{THz}$ frequency range: a high $(\mathrm{H})$ one and low $(\mathrm{L})$ one; these films being 
alternatively and periodically arranged. Depending on the layers properties (refractive index and thickness) and on the periodic arrangement, an incident electromagnetic wave is either transmitted or reflected according to the frequency. The non-transmitted frequency ranges are known as Photonic Band Gap (PBG) and appear periodically in the frequency domain. In first approximation, the spectral position of the $P B G$ depends on the optical thicknesses of the layers, while their depth, known as rejection rate, depends on the refractive index contrast between the alternative and successive layers $\mathrm{H}$ and $\mathrm{L}$ and their number ${ }^{[1]}$. Basically, we optimize the number of the layers to maximize the signal rejection in the PBG, and to limit the losses due to material absorption coefficients. Here, we used pure Low-Density Polyethylene (LDPE) and a mixture of $\mathrm{TiO}_{2}(60 \%)$ - LDPE (40\%), which present refractive indices of $n_{L}=1.51$ and $n_{H}=2.29$, respectively. The tag used in this study has been designed so that the frequency position of the first PBG is centered at $310 \mathrm{GHz}$. In this frequency range, the $\mathrm{THz}$ spectrometer used as the tag reader presents a maximum of dynamics. For that, a stack of 19 alternating layers with respective thicknesses of $\mathrm{e}_{\mathrm{H}}=85 \mu \mathrm{m}$ and $\mathrm{e}_{\mathrm{L}}=240 \mu \mathrm{m}$, has been fabricated (Fig. 1). The information is then contained in the volume of the tag which can be directly incorporated in the product to identify. Let's notice that, playing with the ratio of $\mathrm{TiO}_{2}$ in the mixture, we can precisely tune the refractive index of the effective material from 1.51 (pure polyethylene) up to $2.3\left(60 \% \mathrm{TiO}_{2}-40 \%\right.$ LDPE in mass), that latter value corresponding to a limit from which the material has more mechanical strength. Moreover, the refractive index behavior of the mixture versus the ratio of the $\mathrm{TiO}_{2}$ can be very well predicted using effective medium approaches like the Lichtenecker and Rother formalism ${ }^{[12]}$.A wide range of value of the refractive index can be then chosen either to fabricate the main layers or the structural defects. On other hand, for such a tag family, made of a common polymer material, the cost for this latter depends on the number of layers: about $\$ 0.4$ cts for a 19 layers tag as presented in this study, whereas the manufacturing cost cannot be precisely evaluated has it drastically varies with the number of produced tags. In case of mass-production techniques, the order of magnitude of the cost will be not really different and must be compared to the one of other existing technologies: $\$ 7-\$ 15$ cts for a "classical" RFID tag, or \$0.01- \$0.05 cts in case of $\mathrm{RF}$ tags used in the protection of clothing from theft for example.

\section{CODING PRINCIPLE}

The basic idea is to use the peaks appearing in the PBG when one or several structural defects are introduced in the initial structure of the tag. As the spectral positions and the number of these peaks depend on the structural defects characteristics, they can be used to encode a binary information. We plot in Fig. 2 the transmission coefficient of the tag previously described, around the $1^{\text {st }}$ PBG. Note that the predicted transmission of this multilayer structure (solid line),

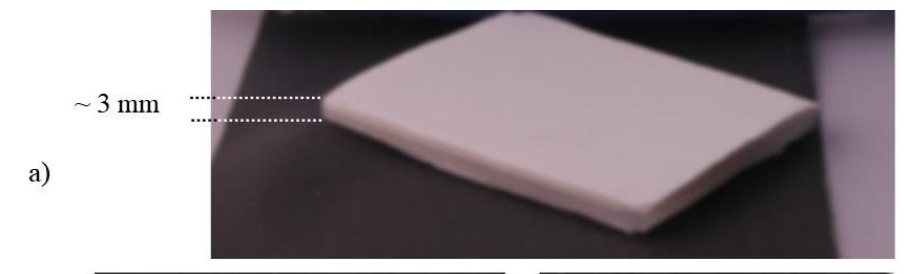

b)
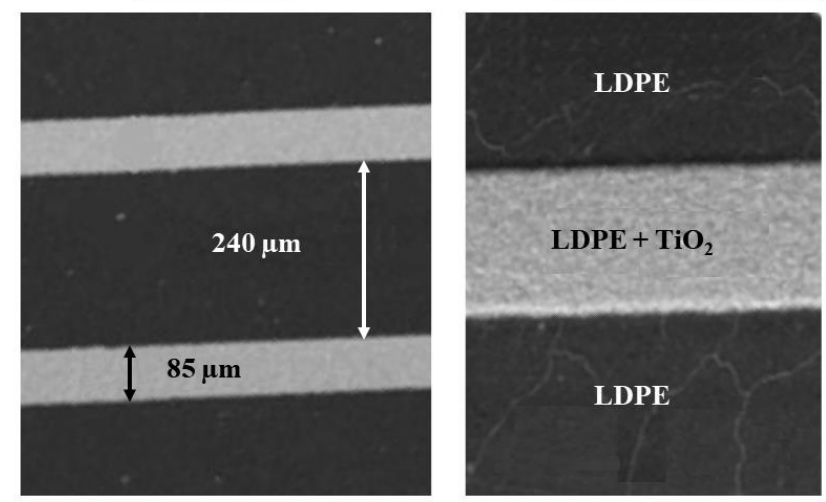

Fig.1 a) Picture of the experimental sample multilayer tag;

b) microscope images of stacked layers made of $\mathrm{LDPE}$ and $\mathrm{TiO}_{2}$.

calculated using the transfer matrix method ${ }^{[13]}$, is in very good agreement with the experiment (circles), and thereby can be used as a prediction tool, to design the tags. The binary information is then encoded by dividing the PBG into sorted channels which are labeled by binary sequences. Some of them are activated by the presence of the peaks to give the unique binary code of the tag. As represented in Fig. 2, in the present case the PBG is divided in $N=8$ channels, each of them corresponding to a 3-bits binary word. Here the channels 3 and 6 are activated by the presence of $M=2$ peaks, therefore, the binary code embedded into the tag spectral response is simply $<010101>$. The coding capacity $\rho(N)$ of the proposed tag is given by numbering the different combinations achievable with $N$ channels. As any combination is obtained by activating from 0 up to $N$ channels, one finally gets the following coding capacity in bits (1):

$$
\rho(N)=\log _{2}\left(\Sigma_{M=0}^{M=N} C_{N}^{M}\right)=N
$$

where the number of channels $N$ depends on the PBG bandwidth $\Delta f$, and the channel width $\delta f$ :

$$
N=\left|\frac{\Delta f}{\delta f}\right|
$$

In our case $N=8$, leading to a coding capacity $\rho(N)$ of about 8 bits. Practically, $\rho(N)$ depends on the reader performances via its frequency resolution and on the tag characteristics. Moreover, the minimum channel width $\delta f$, is imposed by both the tag reader and the spectral width of the defect peaks (depending on the tag losses). $\delta f$ cannot be smaller than twice this latter parameter to ensure affecting a given peak to the right channel. As realistic example, we consider the tag whose spectral response is given in Fig. $2(\Delta f=130 \mathrm{GHz})$ but made of lower absorbing materials $\left(\alpha=1 \mathrm{~cm}^{-1}\right)$. The lower are the 
losses of the material in the layers, the narrower are the peaks induced in the PBG: in that case the full width at half maximum (FWHM) of the peaks is narrowed to about $2 \mathrm{GHz}$ (see Fig. 3). If we consider more a $\mathrm{THz}$ reader presenting a frequency resolution of $100 \mathrm{MHz}$, for example using a continuous waves $(\mathrm{CW}) \mathrm{THz}$ system ${ }^{[13-14]}$, calculations show one could potentially get a coding storage capacity of about 65 bits. Such performances would depend on the measurement quality that is directly linked to the acquisition time. To obtain a sufficient signal-to-noise ratio (SNR) of several $\mathrm{dB}$ which is required reliably identified the tag, we must average the measured signal. In the following section, we focus on the $\mathrm{THz}$ tag performance by considering the evolution of the bit error rate (BER) when the time measurement decreases up to allow a fast identification.

\section{FAST THZ IDENTIFICATION}

In this section we evaluate the ability of a current commercial $\mathrm{THz}$ system to precisely and fastly measure the spectral response of a tag. To ensure an efficient tag reading, the SNR of the $\mathrm{THz}$ system must be sufficient in the considered bandwidth, from $0.25 \mathrm{THz}$ to $0.38 \mathrm{THz}$ in our case. Let's keep in mind that the noise floor is getting higher when averaging is getting lower, therefore the identification process will become hazardous when acquisition time is shortened. To evaluate how fast we can identify a tag with a high reliability, we measure the transmission response of the THID tag using a commercial terahertz time-domain spectroscopy (THz-TDS) system from ADVANTEST ${ }_{\circledast} \quad$ (model TAS7500 SP). Contrarily to conventional THz-TDS systems based on mechanical delay line ${ }^{[15]}$, the pump/probe delay of the TAS7500 SP is achieved using electrically controlled optical sampling (ECOPS) technique ${ }^{[16]}$ that allows very high-speed measurement. The complex transmission coefficient $T(\omega)$ of the tag is calculated from the complex Fourier transforms of the $\mathrm{THz}$ waveform passing through the tag $S(\omega)$, and propagating from the emitter to the receiver in absence of tag, $R(\omega)$ :

$$
T(\omega)=\frac{S(\omega)}{R(\omega)}
$$

The ECOPS-based TAS7500 SP system is a good candidate for fast identification applications, since it allows the record of a transmitted waveform in only $8 \mathrm{~ms}$ over a time window of 132 ps with a time step $\delta t$ of 2 fs. This leads to the acquisition

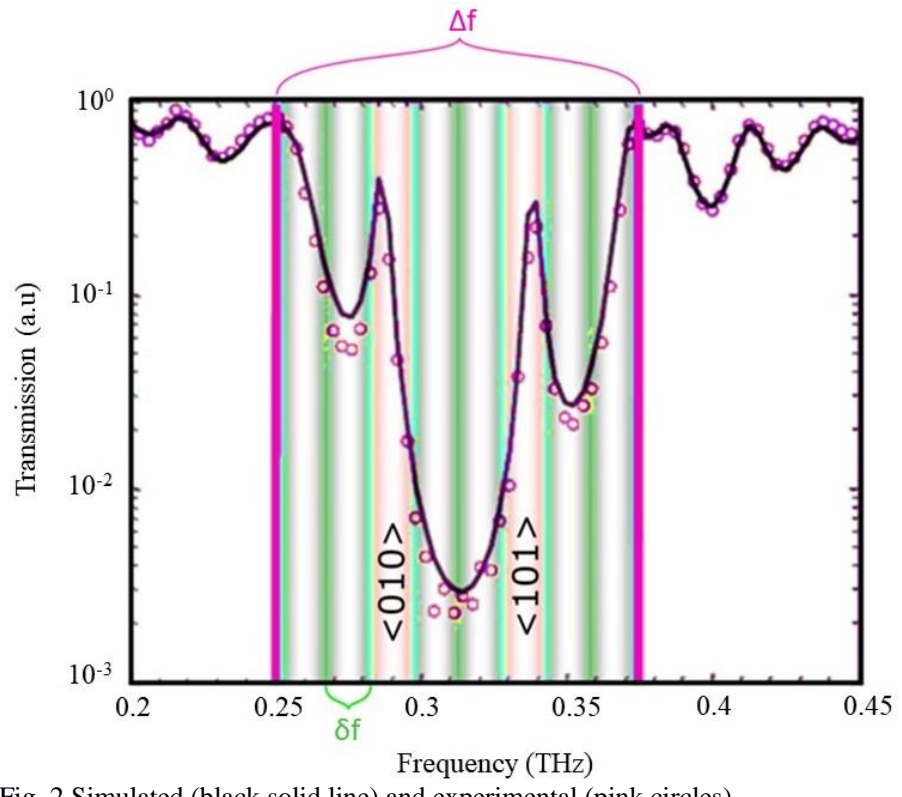

Fig. 2 Simulated (black solid line) and experimental (pink circles) transmission spectra of the proposed tag. The PBG limits are defined by vertical pink lines.

of the tag transmission coefficient over a bandwidth spreading from 0.1 to $4 \mathrm{THz}$ with a spectral resolution of $7.6 \mathrm{GHz}$. This spectral resolution of the reader permits to define $N=8$ channels in the PBG of the tag described above. To estimate the acquisition time that leads to an accurate identification of the tag when using the TAS7500 SP system, we measured the considered tag hundred times in a row with a given averaging time. Then, each of the hundred binary words extracted from the spectral responses and ensured by a homemade software optimized for the peak detection in the spectral range 0.2-0.5 $\mathrm{THz}$, are compared to a reference signature which has been acquired by using the longest averaging time proposed by of the system (few minutes to obtain the reference code $(<010$ $101>)$. Then, the bit error rate (BER) which is directly linked to the identification reliability, is obtained by counting the erroneous binary codes among the hundred measurements, in comparison to the reference one. By repeating the abovedescribed experimental process for different acquisition times, we get Fig. 4 exhibits the BER in function of the acquisition time. Note that for sake of experimental duration, we have deliberately limited the number of measurements to 100 . Consequently, a BER equal to zero means a reliability better than $99 \%$. According to Fig. 4, the BER remains smaller than $1 \%$ for acquisition time as short as $64 \mathrm{~ms}$, leading to a tag identification reliability better than $99 \%$. As expected, the BER is getting greater when acquisition time decreases. 


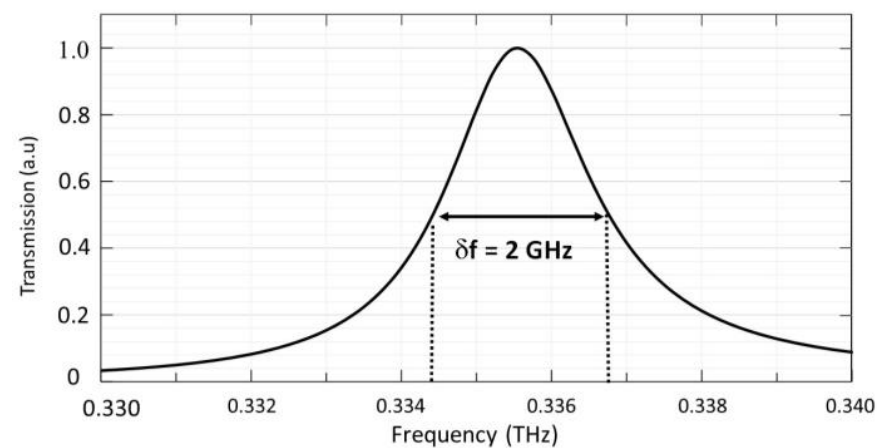

Fig. 3 Simulated peak width, considering a low but realistic absorbing material $\left(1 \mathrm{~cm}^{-1}\right)$ in the layers of the THID tag.

By using the TAS7500 SP system, it is clear that the tag is accurately identified which validate the proposed identification concept. Finally, note that the $\mathrm{THz}$ reader we used in this study is not optimized for identification applications.

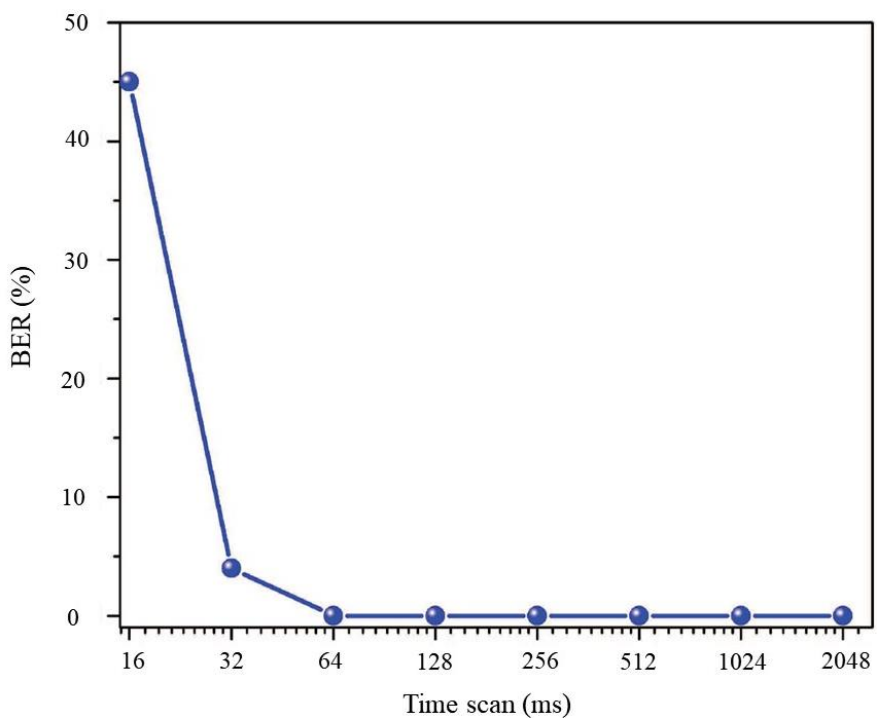

Fig. 4 Variation of the BER obtained using the tag versus the acquisition time.

\section{CONCLUSION}

In this study, we have demonstrated the possibility of a fast identification of a THID tag with a 5 bits binary coding capacity by using a commercial time domain spectrometer

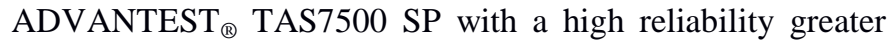
than 99 percent in less than $64 \mathrm{~ms}$. We have obtained such performances by a comparison of binary words that are encoded in the frequency domain using the spectral position of the defect peaks in the PBG of a few mm thick tag. To evaluate the ability of a $\mathrm{THz}$ reader for identification applications, we also must to take into account of the intrinsic noise of the system ${ }^{[17]}$ to ensure a correct identification. More, the recent development of ultrafast high-resolution ASOPSbased THz-TDS systems ${ }^{[18]}$ associated with de-noising extraction techniques ${ }^{[19]}$ would provide powerful $\mathrm{THz}$ reader [20] very promising for the fast identification of tags with coding capacities of several tens of bits. To get these performances, the frequency resolution of the $\mathrm{THz}$ reader must be also improved, to get a greater number of channels, and by increasing the number of peaks in the PBG on the tag point of view $^{[21]}$.

\section{ACKNOWLEDGMENTS}

The authors want to thank the region Auvergne - Rhône Alpes and the University Grenoble Alpes for financing this project through ARC6 research program, and Dr Paul H Dallin and Dr John Andrews from Clairet Scientific Ltd (Northampton, UK) for having put at our disposal the TAS7500 SP spectrometer.

\section{REFERENCES}

[1] M. Bernier, F. Garet, E. Perret, L. Duvillaret, S. Tedjini, "THz encoding approach for secured chipless radio frequency identification," Appl. Opt., vol. 50, no. 23, pp. 4648-4655, 2011.

[2] E. Perret, M. Hamdi, A. Vena, F. Garet, M. Bernier, L. Duvillaret and S Tedjini, "RF and $\mathrm{THz}$ identification using a new generation of chipless RFID tags," Radioengineering - Special Issue towards EuCAP 2012: Emerging Materials, Methods, and Technologies in Antenna \& Propagation, vol. 20, pp. 380-386, 2011.

[3] H. Plötz and K. Nohl, "Analyzing RFID Security," 25th Chaos Communication Congress, Berlin, Germany, Dec. 2008.

[4] F. Brown, "RFID Hacking: Live Free or RFID Hard," Las Vegas, USA, NV Black Hat USA, Las Vegas, USA, Aug. 2013.

[5] H. Welte, "Reverse Engineering a real-world RFID payment system," 27th Chaos Communication Congress, Berlin, Germany, 2010.

[6] D. Cumming, T. Drysdale, "Security label which is optically read by terahertz radiation", US Patent Application, US20060231625A1, 200303-12.

[7] Y. Guan, M. Yamamoto, T. Kitazawa, S. R. Tripathi, K. Takeya, and K Kawase, "A concealed barcode identification system using terahertz time-domain spectroscopy," J. Infrared Millim. Terahertz Waves, vol. 36, no. 3, pp. 298-311, 2015.

[8] Y. Kujime, M. Fujita, and T. Nagatsuma, "Terahertz Tag Using Photonic-Crystal Slabs", IEEE Journal of Lightwave Technology, 2018.

[9] Z. Ali, F. Bonnefoy et al., "Potential of chipless authentication based on randomness inherent in fabrication process for RF and THz", Antennas and Propagation EuCAP, 11th European Conference on (pp. 25592563), 2017

[10] F. Bonnefoy, M. Hamdi et al., "Authentication in the $\mathrm{THz}$ domain: a new tool to fight counterfeiting" $9^{\text {th }} \mathrm{THz}$ days conference, 2017.

[11] Choi, Sung-wook, NA-RI, L.E.E., Chang, Hyun-Joo, et al., "Package damage inspection device", U.S. Patent Application, 15/579,412, 2018 05-24.

[12] M. Hamdi, F. Garet, L. Duvillaret, P. Martinez, and G. E. P. Tourtollet, "Identification tag in the terahertz frequency domain using low-cost and tunable refractive index materials," Ann. Telecommun., vol. 68, no. 7, pp. 415-424, 2013.

[13] H. Němec, L. Duvillaret, F. Quemeneur, and P. Kužel, "Defect modes caused by twinning in one-dimensional photonic crystals," JOSA B, vol 21, no. 3, p. 548, 2004

[14] H. Němec, P. Kuzel, F. Garet and L. Duvillaret, "Time-domain terahertz study of defect formation in one-dimensional photonic crystals," Appl. Opt., vol. 43, no. 9, pp. 1965-1970, 2004. 
[15] P. Y. Han, and X. C. Zhang, "Free-space coherent broadband terahertz time-domain spectroscopy," Measurement Science and Technology, vol. 12, no. 11, 2001.

[16] Y. Kim and D.-S. Yee, "High-speed terahertz time-domain spectroscopy based on electrically controlled optical sampling," Opt. Lett., vol. 35, pp. 3715-3717, 2010.

[17] L. Duvillaret, F. Garet and J.L Coutaz, "Influence of noise on the characterization of materials by terahertz time-domain spectroscopy", JOSA B, 17(3), 452-461, 2000.

[18] A. Bartels, R. Cerna, C. Kistner, A. Thoma, F. Hudert, C. Janke, and T. Dekorsy, "Ultrafast time-domain spectroscopy based on high-speed asynchronous optical sampling," Review of Scientific Instruments, vol. 78, pp. 035107, 2007.

[19] B. Ferguson and D. Abbott, "De-noising techniques for terahertz responses of biological samples," Microelectronics Journal, vol. 32, pp 943-953, 2001

[20] [17] Y. Kim, K. H. Jin, J. C. Ye, J. Ahn, and D.-S. Yee, "Wavelet Power Spectrum Estimation for High-resolution Terahertz Timedomain Spectroscopy," JOSK, vol. 15, no. 1, pp. 103-108, 2011

[21] E. Perret et al., "THID, the next step of chipless RFID", in Proc. IEEE International Conf. on RFID, Penang, Malaysia, pp. 261-268, 2013. 